\title{
On a discrete version of the wave equation
}

\author{
Eszter Gselmann
}

Dedicated to Professor János Aczél on his 90th birthday

\begin{abstract}
The main purpose of this paper is to determine the general (and also the continuous) solutions of the discrete wave equation, that is, to solve the following partial difference equation

$$
\underset{(x)}{\Delta_{1}^{2}} u(x, y)=\underset{(y)}{\Delta_{1}^{2}} u(x, y) .
$$

Mathematics Subject Classification (2010). Primary 39A14; Secondary 43A45, 39B40.

Keywords. partial difference equation, wave equation, exponential polynomial, spectral analysis and synthesis.
\end{abstract}

\section{Introduction}

Numerical partial differential equations is a rather large area of mathematics. There exist many different approaches to solve partial differential equations, the so-called finite difference method is one of them.

This is nothing but a numerical method for approximating the solutions to (ordinary or partial) differential equations using finite difference equations to approximate derivatives. More precisely, the method is the following.

Assume $u$ is a function of the two independent variables $x$ and $y$. Subdivide the $x-y$ plane into sets of equal rectangles of sides $\delta x=h$ and $\delta y=k$, and let the co-ordinates $(x, y)$ of the representative mesh point $P$ be

$$
x=i h \quad \text { and } \quad y=j h \text {, }
$$

where $i, j \in \mathbb{Z}$. Denote the value of $u$ at $P$ by

$$
u_{P}=u(i h, j k)=u_{i, j} .
$$

This research has been supported by the Hungarian Scientific Research Fund (OTKA) Grant NK 81402 and by the TÁMOP 4.2.4.A/2-11-1-2012-0001 (Nemzeti Kiválóság Program - Hazai hallgatói, illetve kutatói személyi támogatást biztosító rendszer kidolgozása és müködtetése konvergencia program) project implemented through the EU and Hungary co-financed by the European Social Fund. 


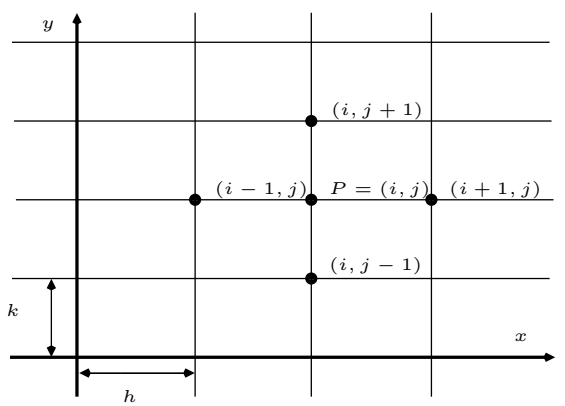

Assuming that $u$ is two times continuously differentiable, due to Taylor's theorem

$$
\left(\frac{\partial^{2} u}{\partial x^{2}}\right)_{P} \approx \frac{u((i+1) h, j k)-2 u(i h, j k)+u((i-1) h, j k)}{h^{2}},
$$

that is,

$$
\left(\frac{\partial^{2} u}{\partial x^{2}}\right)_{P} \approx \frac{u_{i+1, j}-2 u_{i, j}+u_{i-1, j}}{h^{2}}
$$

with a leading error of order $h^{2}$.

Obviously, while applying the finite difference method, partial difference equations arise rather naturally. Among the most important of all hyperbolic partial differential equations is undoubtedly the wave equation, i.e.,

$$
\frac{\partial^{2} u}{\partial t^{2}}=\frac{\partial^{2} u}{\partial x^{2}}
$$

The main aim of this paper is to consider its discrete counterpart, that is, the following partial difference equation

$$
\underset{(x)}{\Delta_{1}^{2} u(x, y)}=\underset{(y)}{\Delta_{1}^{2} u(x, y)}
$$

In other words, we would like to solve equation

$$
u(x+2, y)-2 u(x+1, y)=u(x, y+2)-2 u(x, y+1)
$$

at first on $\mathbb{Z} \times \mathbb{Z}$ and also on $\mathbb{R} \times \mathbb{R}$.

\section{A method based on spectral analysis and synthesis}

In what follows we will shortly summarize a method (based on spectral analysis and synthesis) developed by L. Székelyhidi (see [8,9] and also [4,5]).

Roughly speaking, spectral analysis and synthesis deal with the description of translation invariant function spaces over locally compact Abelian groups. Such type of subspaces naturally arise e.g., in the theory of ordinary and partial differential, resp. difference equations. The main problem is to find an appropriate class of basic functions (they are the so-called exponential monomials). If in addition, their linear hull is dense, we say that spectral synthesis holds for this subspace. 
Let $G$ be a locally compact Abelian group and denote $\mathscr{C}(G)$ the set of all complex valued continuous functions defined on $G$ equipped with the topology of uniform convergence on compact sets. On a variety we mean a proper translation invariant closed subspace of $\mathscr{C}(G)$.

One of the earliest result in the theory of spectral analysis and synthesis is due to L. Schwartz [6].

Theorem 2.1 (Schwartz). In $\mathscr{C}(\mathbb{R})$ any variety is the closed linear hull of exponential monomials which are contained in it.

Let $\mathscr{C}\left(\mathbb{Z}^{n}\right)$ denote the linear space of all complex valued continuous function defined on $\mathbb{Z}^{n}$. Concerning $\mathscr{C}\left(\mathbb{Z}^{n}\right)$ M. Lefranc in 3 proved the following.

Theorem 2.2 (Lefranc). In $\mathscr{C}\left(\mathbb{Z}^{n}\right)$ any variety is the closed linear hull of exponential monomials which are contained in it.

As a consequence of Lefranc's result, the following statement was proved in Székelyhidi 7].

Theorem 2.3. Spectral synthesis holds for any finitely generated discrete Abelian groups.

Let $\Gamma$ be a nonempty set, $c_{\gamma}: \mathbb{Z}^{k} \rightarrow \mathbb{C}$ be a finitely supported function for all $\gamma \in \Gamma$. Then all solutions $f: \mathbb{Z}^{k} \rightarrow \mathbb{C}$ of the system of linear difference equations

$$
\sum_{y \in \mathbb{Z}^{k}} c_{\gamma}(y) f(x+y)=0 \quad\left(x \in \mathbb{Z}^{k}, \gamma \in \Gamma\right)
$$

form a variety, which is proper if and only if at least one of the functions $c_{\gamma}$ are non identically zero.

Theorem 2.4. The spectrum of $(*)$ is the set of all $\lambda \in \mathbb{C}^{k}$ with nonzero components of the system of algebraic equations

$$
\sum_{y \in \mathbb{Z}^{k}} c_{\gamma}(y) f(x+y)=0 \quad\left(x \in \mathbb{Z}^{k}\right) .
$$

Theorem 2.5. The pair $(\lambda, p)$ is in the spectral set of equation $(*)$ and $p$ is a nonzero polynomial, then $\lambda$ is in the spectrum of $(*)$.

Theorem 2.6. The annihilator ideal of

$$
\sum_{y \in \mathbb{Z}^{k}} c_{\gamma}(y) \lambda^{y} p(x+y)=0
$$

is generated by the differential operators

$$
\sum_{y \in \mathbb{Z}^{k}} c_{\gamma}(y) \lambda^{y}\langle y, \partial\rangle^{j}
$$

for all $\gamma \in \Gamma$ and $j=0,1,2, \ldots$ 


\section{Solutions of the discrete wave equation}

Our main results concern the general as well as the regular solutions of the discrete wave equation, that is, our main purpose in this section is solve partial difference equation

$$
u(x+2, y)-2 u(x+1, y)=u(x, y+2)-2 u(x, y+1)
$$

at first on $\mathbb{Z} \times \mathbb{Z}$ and after that on $\mathbb{R} \times \mathbb{R}$.

Theorem 3.1. Assume that for the function $u: \mathbb{Z} \times \mathbb{Z} \rightarrow \mathbb{C}$ functional equation

$$
u(x+2, y)-2 u(x+1, y)=u(x, y+2)-2 u(x, y+1)
$$

is fulfilled for any $(x, y) \in \mathbb{Z} \times \mathbb{Z}$. Then and only then, there exist functions $\alpha, \beta: \mathbb{Z} \rightarrow \mathbb{C}$ such that $\beta$ is a generalized second order polynomial and

$$
u(x, y)=\alpha(x+y)+\beta(x-y) \quad(x, y \in \mathbb{Z}) .
$$

Proof. Due to the method of L. Székelyhidi, presented in the previous section, at first it is enough to determine the exponential polynomial solutions of equation $\mathscr{W}$. The corresponding system of characteristic equations is

$$
\begin{aligned}
{\left[\lambda^{2}\left(2 \cdot \partial_{1}+0 \partial_{2}\right)^{j}-\right.} & \left.2 \lambda\left(1 \cdot \partial_{1}+0 \cdot \partial_{2}\right)^{j}\right] p(x, y) \\
= & {\left[\mu^{2}\left(0 \cdot \partial_{1}+2 \cdot \partial_{2}\right)^{j}-2 \mu\left(0 \cdot \partial_{1}+1 \cdot \partial_{2}\right)^{j}\right] p(x, y) }
\end{aligned}
$$

for any $j=0,1,2, \ldots$ In case $j=0$ we get the algebraic equation

$$
\lambda^{2}-2 \lambda=\mu^{2}-2 \mu,
$$

from which we get that either $\mu=\lambda$ or $\mu=2-\lambda$.

Suppose that $\mu=\lambda$ is fulfilled. Then the above system of equation for $j=1$ yields that

$$
\lambda(\lambda-1) \partial_{1} p(x, y)=\lambda(\lambda-1) \partial_{2} p(x, y)
$$

Again we have two alternatives, either $\lambda \neq 1$ or $\lambda=1$. In case $\lambda \neq 1$, then for the polynomial $p$, we get the partial differential equation

$$
\partial_{1} p(x, y)=\partial_{2}(x, y) \text {. }
$$

From this, we derive that

$$
p(x, y)=\alpha(x+y)
$$

with a certain function $\alpha$.

In case $\lambda=1$, then the characteristic equation with $j=1$ is inexpressive. On the other hand, for $j=2$, we get that

$$
\partial_{1}^{2} p(x, y)=\partial_{2}^{2} p(x, y) \text {. }
$$

This yields however that

$$
p(x, y)=\alpha(x+y)+\beta(x-y)
$$

is fulfilled with certain functions $\alpha$ and $\beta$. 
Suppose finally, that $\mu=2-\lambda$ is satisfied. In this case the system a characteristic equations with $j=1$ means that

$$
\lambda(\lambda-1) \partial_{1} p(x, y)=(\lambda-2)(\lambda-1) p(x, y) .
$$

Clearly, $\lambda \neq 2$, otherwise $\mu$ would be zero and we are only interested in the nontrivial exponential polynomial solutions. If so, we get after some rearrangement that

$$
\lambda \partial_{1} p(x, y)=(\lambda-1) \partial_{2} p(x, y)
$$

that is,

$$
p(x, y)=\alpha((\lambda-1) x+\lambda y)
$$

holds with a certain function $\alpha$. Furthermore, the system of characteristic equations for $j=2$ is

$$
\left(2 \lambda^{2}-\lambda\right) \partial_{1}^{2}=\left(2 \lambda^{2}-9 \lambda+10\right) \partial_{2}^{2}
$$

Inserting the above form of $p$ into this, we get that

$$
\left(2 \lambda^{2}-\lambda\right) \cdot(\lambda-1)^{2} p(x, y)=\left(2 \lambda^{2}-9 \lambda+10\right) \cdot \lambda^{2} p(x, y) .
$$

Therefore, either $p$ is identically zero or $\lambda$ is a solution of the algebraic equation

$$
\left(2 \lambda^{2}-\lambda\right) \cdot(\lambda-1)^{2}=\left(2 \lambda^{2}-9 \lambda+10\right) \cdot \lambda^{2} .
$$

The solutions of this equation are

$$
\lambda_{1}=0 \quad \lambda_{2}=\frac{3+\sqrt{13}}{4} \quad \lambda_{3}=\frac{3-\sqrt{13}}{4} .
$$

Clearly, the only interesting cases are $\lambda_{2,3}=\frac{3 \pm \sqrt{13}}{4}$. For $j=3$ the system of characteristic equations is

$$
\left(8 \lambda^{2}-2 \lambda\right) \partial_{1}^{3} p(x, y)=\left(8(2-\lambda)^{2}-2(2-\lambda)\right) \partial 2^{3} p(x, y)
$$

that is,

$$
\left(8 \lambda^{2}-2 \lambda\right) \cdot(\lambda-1)^{3} p(x, y)=\left(8(2-\lambda)^{2}-2(2-\lambda)\right) \cdot \lambda^{3} p(x, y)
$$

has to be fulfilled. This however means that either $\lambda$ is a solution of the algebraic equation

$$
\left(8 \lambda^{2}-2 \lambda\right) \cdot(\lambda-1)^{3}=\left(8(2-\lambda)^{2}-2(2-\lambda)\right) \cdot \lambda^{3}
$$

or $p$ is identically zero. It is not too hard to see (after a simple substitution) that neither $\lambda_{2}$ nor $\lambda_{3}$ fulfills this equation.

All in all, the exponential polynomial form solution of equation $\sqrt[W]{ }$ are

$$
u(x, y)=\alpha(x+y)+\beta(x-y) .
$$

However, substituting this form into $\mathscr{W}$ we get that $\beta$ must be a generalized second order polynomial.

The pointwise limits of linear combinations of such functions are also of the form

$$
u(x, y)=\alpha(x+y)+\beta(x-y) \quad(x, y \in \mathbb{Z}),
$$


where $\alpha: \mathbb{Z} \rightarrow \mathbb{C}$ is any function and $\beta: \mathbb{Z} \rightarrow \mathbb{C}$ is a generalized second order polynomial.

Conversely, an easy computation shows that the above function satisfies equation $\mathscr{W}$ for arbitrary $(x, y) \in \mathbb{Z} \times \mathbb{Z}$.

Theorem 3.2. Suppose that the function $u: \mathbb{R} \times \mathbb{R} \rightarrow \mathbb{C}$ satisfies functional equation

$$
u(x+2, y)-2 u(x+1, y)=u(x, y+2)-2 u(x, y+1)
$$

for all $(x, y) \in \mathbb{R} \times \mathbb{R}$. Then and only then, there exist functions $\alpha, \beta: \mathbb{R} \rightarrow \mathbb{C}$ such that $\beta$ is a generalized second order polynomial, further, there is an antisymmetric and biadditive function $B: \mathbb{R} \times \mathbb{R} \rightarrow \mathbb{C}$ such that

$$
u(x, y)=\alpha(x+y)+\beta(x-y)+B(x, y) \quad(x, y \in \mathbb{R})
$$

is fulfilled.

Proof. After a similar argument that was presented in the proof of Theorem 3.1 , we get that the exponential polynomial solution of equation $\sqrt[W]{ }$ on $\mathbb{R} \times \mathbb{R}$ are of the form

$$
p(x, y)=\alpha(x+y)+\beta(x-y),
$$

where $\beta$ is a generalized second order polynomial. The pointwise limits of linear combinations of such functions also has this form. Additionally, the mapping

$$
\mathbb{R} \times \mathbb{R} \ni(x, y) \longmapsto u(x, y)-u(x, 0)-u(y, 0)
$$

fulfills the so-called cocycle equation, that is

$$
F(x+y, z)+F(x, y)=F(x, y+z)+F(y, z) \quad(x, y, z \in \mathbb{R}) .
$$

Thus applying a result of Hosszú [2] finally we get that

$$
u(x, y)=\alpha(x+y)+\beta(x-y)+B(x, y) \quad(x, y \in \mathbb{R}),
$$

with an appropriate function $\alpha: \mathbb{R} \rightarrow \mathbb{C}$, a generalized second order polynomial $\beta: \mathbb{R} \rightarrow \mathbb{C}$ and an antisymmetric and biadditive function $B: \mathbb{R} \times \mathbb{R} \rightarrow \mathbb{C}$. The converse is an easy computation.

In view of the results of the previous theorem we are also able to determine the continuous solutions of equation $\sqrt[W]{ }$ that is contained in the following.

Corollary 3.3. Let us assume that the continuous function $u: \mathbb{R} \times \mathbb{R} \rightarrow \mathbb{C}$ satisfies functional equation

$$
u(x+2, y)-2 u(x+1, y)=u(x, y+2)-2 u(x, y+1)
$$

for all $(x, y) \in \mathbb{R} \times \mathbb{R}$. Then and only then, there exists a continuous function $\alpha: \mathbb{R} \rightarrow \mathbb{C}$ and constants $a, b, c$ such that

$$
u(x, y)=\alpha(x+y)+a(x-y)^{2}+b(x-y)+c \quad(x, y \in \mathbb{R})
$$

holds. 
In [1] J. A. Baker investigated a partial difference equation rather similar to equation $\mathscr{W}$. Namely, in the aforementioned paper he presented the continuous solutions of equation

$$
u(x+h, y)-2 u(x+h, y)=u(x, y+h)-2 u(x, y+h) \quad(x, y, h \in \mathbb{R}),
$$

where $u: \mathbb{R} \times \mathbb{R} \rightarrow \mathbb{C}$ is the unknown function. We would like to remark that in view of Theorem 3.2 we can determine not only the continuous but also the general solutions of this equation. To see this, it is enough to substitute $h=1$ and apply Theorem 3.2 .

Corollary 3.4. Assume that the function $u: \mathbb{R} \times \mathbb{R} \rightarrow \mathbb{C}$ fulfills functional equation

$$
u(x+h, y)-2 u(x+h, y)=u(x, y+h)-2 u(x, y+h)
$$

for all $x, y, h \in \mathbb{R}$. Then and only then, there exists functions $\alpha, \beta: \mathbb{R} \rightarrow \mathbb{C}$ such that $\beta$ is a generalized second order polynomial, further, there is an antisymmetric and biadditive function $B: \mathbb{R} \times \mathbb{R} \rightarrow \mathbb{C}$ such that

$$
u(x, y)=\alpha(x+y)+\beta(x-y)+B(x, y) \quad(x, y \in \mathbb{R})
$$

is fulfilled.

\section{References}

[1] J. A. Baker, An analogue of the wave equation and certain related functional equations, Canad. Math. Bull. 12 (1969), 837-846. MR 0254455 (40 \#7663)

[2] M. Hosszú, On the functional equation $F(x+y, z)+F(x, y)=F(x, y+z)+$ $F(y, z)$, Period. Math. Hungar. 1 (1971), no. 3, 213-216. MR 0289991 (44 \#7176)

[3] M. Lefranc, Analyse spectrale sur $Z_{n}$, C. R. Acad. Sci. Paris 246 (1958), 19511953. MR 0098951 (20 \#5396)

[4] P. K. Sahoo and L. Székelyhidi, A functional equation on $Z_{n} \oplus Z_{n}$, Acta Math. Hungar. 94 (2002), no. 1-2, 93-98. MR 1905789 (2003f:39086)

[5] $\quad$ On the general solution of a functional equation on $\mathbb{Z} \oplus \mathbb{Z}$, Arch. Math. (Basel) 81 (2003), no. 2, 233-239. MR 2009566 (2004h:39063)

[6] L. Schwartz, Sur une propriété de synthèse spectrale dans les groupes non compacts, C. R. Acad. Sci. Paris 227 (1948), 424-426. MR 0027096 (10,249e)

[7] L. Székelyhidi, On discrete spectral synthesis, Functional equations-results and advances, Adv. Math. (Dordr.), vol. 3, Kluwer Acad. Publ., Dordrecht, 2002, pp. 263-274. MR 1912720 (2003g:43006)

[8] _ Difference equations via spectral synthesis, Ann. Univ. Sci. Budapest. Sect. Comput. 24 (2004), 3-14. MR 2168033 (2007j:43006)

[9] _ Discrete spectral synthesis and its applications, Springer Monographs in Mathematics, Springer, Dordrecht, 2006. MR 2279454 (2009d:43001) 
E. Gselmann

Eszter Gselmann

Department of Analysis

Institute of Mathematics

University of Debrecen

P. O. Box: 12 .

Debrecen

H-4010

Hungary

e-mail: gselmann@science.unideb.hu

Submitted: 28 February, 2014. 